\title{
Analytical Solution to Settlement of Cast-In-Situ Thin-Wall Concrete Pipe Pile Composite Foundation
}

\author{
Han-long Liu ${ }^{1,2}$, An Deng ${ }^{1,2}$, and Shi-qing Wen ${ }^{1,2}$ \\ ${ }^{1}$ Key Laboratory for Geotechnical Engineering of Ministry of Water Resource, \\ Hohai University, Nanjing 210098, P.R. China \\ ${ }^{2}$ Geotechnical Research Institute, Hohai University, Nanjing 210098, P.R. China \\ \{hliu,a_deng, sqw\}@hhu.edu.cn
}

\begin{abstract}
The cast-in-situ thin-wall concrete pipe pile (PCC pile) is increasingly employed as a soft soil improvement technique in China. The formed composite foundation by using PCC pile renders a competitive advantage compared to conventional comparable soft soil treatment techniques. To calculate the settlement of PCC composite foundation helps better design a soil improvement scenario and understand the mechanism of PCC composite foundation. In this study, an elastic analytical solution to deformation modulus of PCC composite foundation was developed based upon the pile-soil-cushion interaction system and model analyses. Using the calculated modulus, formula presenting pile-soil stress ratio and composite foundation settlement were derived. A case study was conducted to verify the calculations. It is indicated that calculated settlements are in consistency with fielding monitoring data.
\end{abstract}

Keywords: PCC pile, composite foundation, deformation modulus, pile-soil stress ratio, settlement.

\section{Introduction}

As the drastic developments along the east China areas, a number of infrastructure works, e.g. ports, highway, bridges and warehouses, are constructed or planned. One of the obstacles in constructions is the poor bearing capacity of soft ground, which widely exists in east China, in particular in the vicinities of sea, rivers and lakes. Soil treatment has to be applied to improve the bearing capacity and decrease the settlement of soft soil foundations. The cast-in-situ, thin-wall, concrete pipe pile (PCC pile), a patented technique for soft soil improvement, ${ }^{[1,2]}$ has been extensively employed to address the soft soil concerns.

The PCC pile is a cast-in-situ, thin-wall, concrete pipe pile. The external diameter varies from 1000 to $1500 \mathrm{~mm}$, and the wall thickness is in the range of 150 to 200 $\mathrm{mm}$. The length of PCC pile is decided by ground properties and engineering goals, generally ranging from several meters to tens of meters. PCC piles can either be driven into ground fully as a composite foundation, or be driven partially with embedded reinforcement to set as a retaining structure. Fresh concrete is cast and vibrated at continuous steps to form the pile body. Using PCC composite foundation to improve soft soils has advantages spanning multiple aspects, i.e. concept, construction, performance, and economics. In concept, it maximizes the utilization of 
shaft frictions through both inside and outside soil masses. It also minimizes concrete consumption by converting a solid pile into an economical annular cross section. The dimensions of PCC pile are adjustable, which makes PCC suitable for various soil properties and engineering goals. A steel pipe mold is driven into the soil mass to form an annular-shape room and the fresh concrete is poured. In this manner, the completeness and continuity of pile body is ensured. Since the shaft friction resistance is basically doubled, and the inner and outer soils may co-work together with PCC piles, the bearing capacity of PCC composite foundation is increased in a more effective way than other conventional techniques, e.g. deep-soil-mixing columns.

Mechanical mechanism of PCC composite foundation has not been clearly recognized. As a newly patented technique, the soil-pile interaction and composite foundation settlement are not yet understood. The primary settlement of composite foundation is not easy to estimate. Current applications are basically fulfilled at an empirical basis. Lack of deeper technical knowledge about PCC composite foundation barriers its optimization in designs. To upgrade this technique is also delayed without knowing its mechanical mechanism. This paper presents the establishment of a theoretical frame and computing methods regarding the deformation modulus, the pile-soil stress ratio and the settlement the composite foundation. A case study is conducted to verify the reliability of the derivation and computation.

\section{Method and Analyses}

\subsection{Composite Foundation Model}

A PCC composite foundation model, the pile-soil-cushion unit, is demonstrated in Fig. 1. The upper load $P$ is uniformly applied over the cushion. The cushion is constructed with the thickness of $h_{c}$ and modulus of $E_{c}$. The pile length is $h$, with its outer diameter $D$ and inner diameter $d$. The pile body has elastic modulus $E_{p}$. The elastic modulus of soil layer $i$ within the improvement area is $E_{s i}$. After the adjustment of layer, the uniform load on PCC pile is $P_{s}$. The total soil compression is composed of two parts: $S_{1}$ for $h$ thick improved deposit and $S_{2}$ for underlying soil deposits. The pile compression is $S_{3}$. The settlement of pile tip is $\delta_{p}$. It is known that $\delta_{p}$ is equal to the addition of $S_{1}, S_{2}$ and $S_{3}$.

Assumptions are made with respect to the PCC composite foundation. It is assumed that the composite foundation is an elastic semi-infinite body, ignoring the boundary conditions. The replacement ratio of PCC composite foundation is $m$. PCC piles are arranged at the same spacing. The contact stresses upon cushions, pile heads and soil surface are uniformly distributed. Inner soils' bearing capacity is ignored. Soil-pile composite foundation is homogeneously layered. The underlying soil layers are compressible homogenous elastic body conforming to Hooker's law. The elastic soil layer, outside and inside PCC piles, has identical compression modulus $E_{S}$ and shaft frictional resistance coefficient $K$. When the pile-soil relative displacement $\delta$ is less than the ultimate displacement $\delta_{u}$, the frictional resistance is the product of relative displacement $\delta$ and frictional resistance coefficient $K$. Otherwise, when relative displacement $\delta$ exceeds the ultimate displacement $\delta_{u}$, the frictional resistance is the product of $\delta_{u}$ and $K$. 


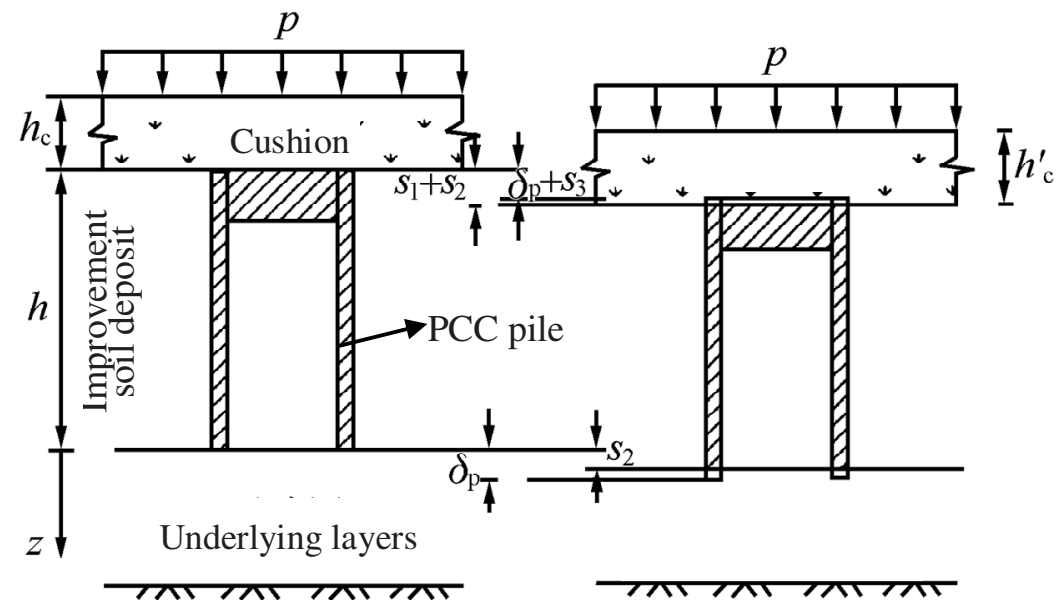

Fig. 1. PCC composite foundation loads and displacements model

\subsection{Model Analyses}

The upper load $P$ on cushion is transferred to pile at $P_{p}$ and soil at $P_{s}$, respectively, and quantified by Eq. 1 according to the pile-soil replacement ratio $m .^{[3]}$

$$
p=m P_{p}+(1-m) P_{s} .
$$

The cushion deformation above pile tip due to $P_{p}$ is expressed as $h_{c}-\left[h_{c}^{\prime}-\left(S_{1}+S_{2}-S_{3}-\delta_{P}\right)\right]$ according to Fig. 1 . Thus, the pile load $P_{p}$ is computed using Eq. 2.

$$
P_{p}=E_{c} \frac{h_{c}-\left[h_{c}^{\prime}-\left(S_{1}+S_{2}-S_{3}-\delta_{P}\right)\right]}{h_{c}} .
$$

On the other hand, the cushion deformation above soil due to $P_{s}$ is expressed as $h_{c}-h_{c}^{\prime}$. The load on soil, $P_{s}$, is shown in Eq. 3.

$$
P_{S}=E_{c} \frac{h_{c}-h_{c}^{\prime}}{h_{c}} .
$$

The stress transferring model of PCC pile in a linear elastic condition is presented in Fig. 2 as recommended for a conventional pile. ${ }^{[4,5]}$ The pile is divided into a number of identical small units. Because all units follow deformation and stress coordination, Eq. 4 is derived and converted into Eq. 5.

$$
\begin{gathered}
N_{z}=N_{z}+\frac{d N_{z}}{d z} d z+\left(f_{z 1} c_{1}+f_{z 2} c_{2}\right) d z . \\
\frac{d N_{z}}{d z}+\left(f_{z 1} c_{1}+f_{z 2} c_{2}\right)=0 .
\end{gathered}
$$



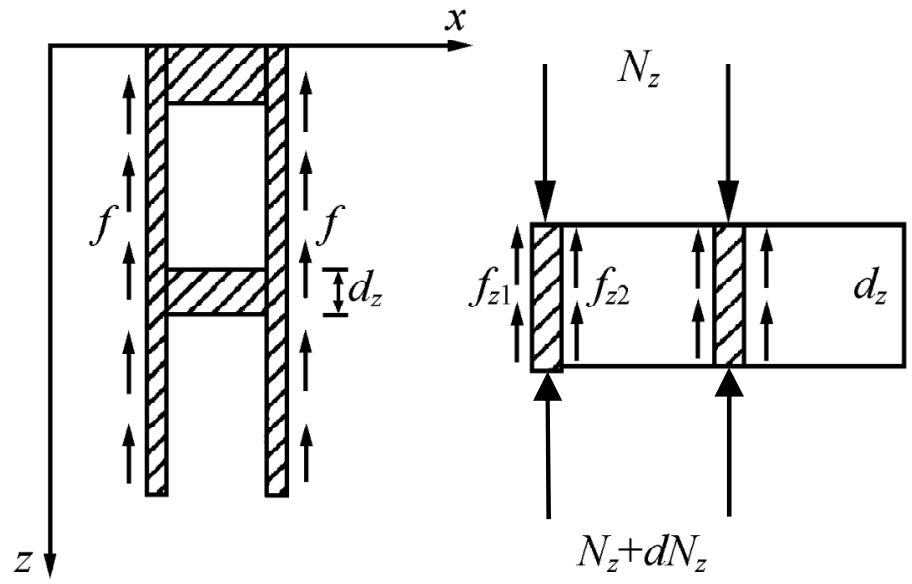

Fig. 2. Linear elastic stress transferring model of PCC pile

where, $f_{z l}$ indicates the outer friction resistance of a unit; $c_{1}$ indicates pile outer perimeter; $f z_{2}$ is the inner friction resistance of a unit; $c_{2}$ is pile inner perimeter.

As it is assumed that soil-pile composite foundation is homogeneously layered, the compression of improved soil deposit, $S_{l}$, can be calculated by using layer-wise summation method as shown in Eq. 6.

$$
S_{1}=\sum_{i=1}^{n} \frac{P_{s i}}{\overline{E_{s i}}} h_{i}=\varphi P_{s} .
$$

where, $n$ is the total layer number of improved soil deposit. The additional stress for layer $i$ is $P_{s i}$. The thickness of layer $i$ is $h_{i}$. The composite modulus of layer $i$ is $E_{s i}$. $\varphi$ represents the equivalent compression coefficient of improved deposit.

Pile may punch into cushion and underlying layer at deep $S_{h}$ and $S_{l}$, respectively. ${ }^{[6]}$ The pile punching effect and displacement coordination between pile and soil are taken in consideration to calculate the composite foundation modulus $\bar{E}_{c}$ as shown in Eq. 7.

$$
\bar{E}_{c} \overline{\mathcal{E}}_{c}=m \varepsilon_{p} E_{p}+(1-m) E_{s} \varepsilon_{s} .
$$

where, $\bar{\varepsilon}_{c}=\varepsilon_{s}=S_{1} / h, \varepsilon_{p}=\left[S_{1}-\left(S_{h}+S_{l}\right)\right] / h \times \varepsilon_{p} . \varepsilon_{p}$ and $\varepsilon_{s}$ represent the vertical strains of pile and soil, respectively. $\bar{\varepsilon}_{c}$ is the vertical strain of composite foundation. $S_{h}$, the depth of upper pile punching, is equal to $\left(S_{1}+S_{2}-\delta_{P}-S_{3}\right) ; S_{l}$, the depth of lower punching, is equal to $\left(\delta_{p}-S_{2}\right)$. Eq. 7 is further converted into Eq. 8.

$$
\bar{E}_{c}=m\left[1-\left(S_{h}+S_{l}\right) / S_{1}\right] E_{p}+(1-m) E_{s}=u_{p} m E_{P}+(1-m) E .
$$


where, $u_{p}=1-\left(S_{h}+S_{l}\right) / S_{1}=S_{3} / S_{1}$, which indicates the performance coefficient of the pile modulus. Its value can be obtained by using iteration method.

The compression of underlying layers, $S_{2}$, can be calculated by using layer-wise summation method as shown in Eq. 9.

$$
S_{2}=\sum_{j=1}^{k} \frac{P_{s j}}{E_{s j}} h_{j} .
$$

where, $k$ is the total number of compressible underlying layers; $P_{s j}$ is the additional stress for underlying layer $j ; h_{j}$ is the thickness of underlying layer $j ; E_{s j}$ is the compression modulus of underlying layer $j$. $P_{s j}$ can be calculated by using stress diffusion method. ${ }^{[3]}$

The total amount of PCC pile compression is relatively small, which can be approximately calculated using Eq. 10.

$$
S_{3}=\left(P_{P} A_{1}\right) /\left(E_{P} A\right)=G P_{P} .
$$

where, $A_{1}=\pi D^{2}$, indicating pile-soil section area; $A=\pi\left(D^{2}-d^{2}\right)$, indicating PCC pile annular area; $G=\left(A_{1} h\right) /\left(E_{P} A\right)$.

Under elastic conditions, the pile-soil displacement is relatively small. Suppose the compression curve of improved deposit is linear, and the compression of rigid piles varies linearly with depth, the outer shaft friction resistance of the pile is expressed using Eq. 11.

$$
f_{z 1}=K\left[\delta_{p}-\left(S_{1}+S_{2}-S_{3}\right)+(z / h)\left(S_{1}-S_{3}\right)\right]
$$

Similarly, suppose the compression curve of the inner soil is linear, the inner shaft friction resistance at point $Z$ is expressed in Eq. 12.

$$
f_{z 2}=K(z / h)\left[\delta_{p}+S_{3}(1-z / h)-S_{2}\right] .
$$

By applying Eqs. 11 and 12 to Eq. 5, Eq. 13 is obtained.

$$
\frac{d N_{Z}}{d Z}=-K c_{1} \frac{S_{1}-S_{3}}{h} z-K c_{1} \delta_{p}+K c_{1}\left(S_{1}+S_{2}-S_{3}\right)-K c_{2} \frac{z}{h}\left(\delta_{p}+S_{3}-S_{2}\right)+K c_{2} S_{3} \frac{z^{2}}{h^{2}} .
$$

Given the boundary condition at pile cap, $N_{z}=P_{p} A_{l}$, as shown in Fig. 2, Eq. 13 is integrated into Eq. 14.

$$
\begin{aligned}
& N_{z}=\frac{1}{3} K c_{2} S_{3} \frac{z^{2}}{h_{2}}-\frac{1}{2} K c_{1} \frac{S_{1}-S_{3}}{h} z^{2}-\frac{1}{2} K c_{2} \frac{z^{2}}{h}\left(\delta_{p}+S_{3}-S_{2}\right)-K c_{1} \delta_{p} z \\
& +K c_{1}\left(S_{1}+S_{2}-S_{3}\right) z+P_{p} A
\end{aligned}
$$

It is supposed that the pile tip foundation conforms to Winkler's Law. ${ }^{[7]}$ Therefore, when point $Z$ reaches to depth $h$ in Fig. 2, the axial stress of pile tip, $N_{z}$, is $k_{s} A\left(\delta_{P^{-}} \delta_{2}\right)$. $K_{s}$ is the coefficient of subgrade reaction for the pile tip bearing layer. Apply it to Eq. 14, Eq. 15 is obtained. 


$$
\frac{1}{2} K c_{1} h\left(S_{1}-S_{3}\right)-\frac{1}{6} K c_{2} h S_{3}-\alpha \delta_{P}+\alpha S_{2}+P_{p} A_{1}=0 .
$$

where, $\alpha=k_{s} A+K c_{1} h+\frac{1}{2} K c_{2} h$.

Combining Eqs. 1 to 3, 6, 10 and 15, pile load $P_{p}$, soil load $P_{s}$, and compressed cushion thickness $h_{c}^{\prime}$ are computed as shown in Eqs. 16, 17 and 18, respectively.

$$
\begin{gathered}
P_{p}=\frac{P[(1-m) \theta \varphi+\alpha \beta]}{m \alpha \beta+(1-m)\left(\theta G+\alpha \beta+A_{1}-\frac{1}{6} K c_{2} h G\right)} . \\
P_{s}=\frac{P\left(\theta G+\alpha \beta+A_{1}-\frac{1}{6} K c_{1} h G-m \theta \varphi\right.}{m \alpha \beta+(1-m)\left(\theta G+\alpha \beta+A_{1}-\frac{1}{6} K c_{2} h G\right)} . \\
h_{c}=\left(1-\frac{P_{s}}{E_{c}}\right) h_{c}=h_{c}-P_{s} \beta .
\end{gathered}
$$

where, $\beta=h_{c} / E_{c}, \theta=k_{s} A+\frac{1}{2} K c_{1} h+\frac{1}{2} K c_{2} h+\frac{1}{2} K c_{2} h$.

Thus, the total deformation of the composite foundation is shown in Eq. 19.

$$
S=S_{1}+S_{2}+h_{c}-h_{c}^{\prime}=\varphi P+\beta P_{s}+S_{2} .
$$

The pile-soil stress ratio is shown in Eq. 20.

$$
N=\frac{P_{p}}{P_{o}}=\frac{(1-m) \theta \varphi+\beta \alpha}{\theta G+\alpha \beta+A_{1}-\frac{1}{6} K c_{2} h G-m \theta \varphi}
$$

Parameter $u_{p}$ can also be calculated using Eq. 21 .

$$
u_{P}=\frac{S_{1}}{S_{3}}=\frac{G}{\varphi} \frac{(1-m) \theta \varphi+\alpha \beta}{m \alpha \beta+(1-m)\left(\theta G+\alpha \beta+A_{1}-\frac{1}{6} K c_{2} h G\right)} .
$$

In Eq. 21 , because $\varphi$ is related to $u_{p}$, value of $u_{p}$ cannot be calculated directly. Iteration method may provide an analytical value of $u_{p}$ by following procedures.

First, suppose a relatively small value of $u_{p 1}$ and run the calculations to obtain the value of $\varphi$ and a new value $u_{p 2}$. Secondly, run a comparison between calculated $u_{p 2}$ and supposed value $u_{p 1}$. If $u_{p 2}$ is larger than $u_{p 1}$, increase supposed $u_{p 1}$ in a proper addition to $u_{p 3}$, re-run the calculations. Repeat the procedures until $u_{p i}$ and 
$u_{p(i-1)}$ are identical. Final value $u_{p i}$ is the performance coefficient of pile modulus. Based on the iteratively inferred $u_{p i}$ value, the pile-soil stress ratio and the PCC composite foundation settlement are obtained. A program can be coded to run the iterative computations according to design data of PCC composite foundation.

\section{Case Study}

A case study was conducted to verify the analytical solution developed in this study. Hang-Ning Highway links Hangzhou and Nanjing and crosses most soft ground zones. The PCC composite foundation was applied for improving soils at Changxing section of the highway.

The PCC pile spacing is $2.5 \mathrm{~m}$. The outer diameter $D$ and inner diameter $d$ of the pile is 1.0 and $0.76 \mathrm{~m}$, respectively. The pile length $h$ is $16 \mathrm{~m}$. The composite foundation displacement ratio $m$ is 0.053 . The cushion, composed of gravels and sands, is $0.5 \mathrm{~m}$ thick with $E_{c}$ being $31 \mathrm{MPa}{ }^{[8]}$ The coefficient subgrade reaction $K_{s}$ is $20 \mathrm{MPa} \cdot{ }^{[8]}$ The embankment filling height is $4.5 \mathrm{~m}$. After iterative computations, $U_{p}$ is calculated as 5.6\%; pile-soil stress ratio is 12.2; the settlement of improvement deposit $S_{1}$ is $21.5 \mathrm{~mm}$; the settlement of underlying layers $S_{2}$ is $104.3 \mathrm{~mm}$; the total settlement of composite foundation is $125 \mathrm{~mm}$. Field monitoring data indicate that the maximum pile-soil stress ratio is 16 , and the composite foundation settlement is 141 $\mathrm{mm}$. Calculated results are less than but close to monitored data. Therefore, analytical solution is validated.

\section{Concluding Remarks}

Analytical solutions are derived regarding the composite modulus, the pile-soil stress ratio, and the settlement of PCC composite foundation, based on the displacement coordination of pile-soil-cushion unit model. The analytical solutions help understand the pile-soil interaction mechanism, optimize PCC composite foundation design, and predict foundation's future primary settlement. Monitored data in a highway project using PCC composite foundation present good agreement with calculated data according to analytical solutions, and thus the derived solutions are verified.

Acknowledgment. The study was supported by a grant of National Natural Science Foundation of China (No. 50679017).

\section{References}

1. Liu, H. L., Fei, K., Ma, X. H.: Field Pour Concrete Cased Pile with Vibrated and Steel Tube Mould Technology and its Application I: Development and Design Theory. Rock Soil Mech. 24 (2003) 164-168

2. Liu, H. L., Hao X. Y., Fei, K.: Field Pour Concrete Cased Pile with Vibrated Steel Tube Mould Technology and its Application II: Application and in-situ Test. Rock Soil Mech. 24 (2003) 372-375 
3. Gong, X. N.: The Theory and Practice of Composite Foundation. China Architecture Building Press, Beijing (2001)

4. Zhang, S. N.: Pile-Soil Stress Ratio Study of Mixing Pile Composite Foundation. Foundation Treatment, 6 (1995) 9-14

5. Ronald Y. S. P., Feng, J.: Rational Mechanical of Axial Soil-Pile Interaction. J. Eng. Mech. 119 (1993) 813-831

6. Mao, Q., Gong, X. N.: Research on the Properties of Cushion of the Composite Ground. Rock Soil Mech. 19 (1998) 67-73

7. Fu, J. H., Song, E. X.: Analysis of Rigid Pile Composite Foundation Working Performance. Rock Soil Mech. 21 (2000) 335-339

8. Zhou, J. X., Wang H. J., Yu, S. M.: Foundation Construction. Tsinghua University Press, Beijing (1996) 